\title{
Numerical solution of high-order linear integro- differential equations with variable coefficients using two proposed schemes for rational Chebyshev functions
}

\author{
Mohamed Ramadan ${ }^{1}$, Kamal Raslan ${ }^{2}$, Adel Hadhoud ${ }^{3}$ and Mahmoud Nassar ${ }^{4}$ \\ ${ }^{1}$ Mathematics Department, Faculty of Science, Menoufia University, Egypt \\ ${ }^{2,4}$ Mathematics Department, Faculty of Science, Al-Azhar University, Cairo, Egypt \\ ${ }^{3}$ Higher Institute of Engineering and Technology in Mansoura, Egypt
}

Received: 24 November 2015, Accepted: 21 January 2016

Published online: 19 June 2016.

\begin{abstract}
In this paper, a rational Chebyshev (RC) collocation method is presented to solve high-order linear Fredholm integrodifferential equations with variable coefficients under the mixed conditions, in terms of RC functions by two proposed schemes. The proposed method converts the integral equation and its conditions to matrix equations, by means of collocation points on the semiinfinite interval, which corresponding to systems of linear algebraic equations in RC coefficients unknowns. Thus, by solving the matrix equation, RC coefficients are obtained and hence the approximate solution is expressed in terms of RC functions. Numerical examples are given to illustrate the validity and applicability of the method. The proposed method numerically compared with others existing methods as well as the exact solutions where it maintains better accuracy.
\end{abstract}

Keywords: Rational Chebyshev functions, Collocation method, Fredholm integro-differential equations.

\section{Introduction}

In recent years, the studies of mixed integro-differential equations are developed very rapidly and intensively. Integro-differential equation is an equation that the unknown function appears under the sign of integration and it also contains the derivatives and functional arguments of the unknown function. It can be classified to Fredholm integro-differential equations and Volterra integro-differential equations. In this paper we focus on Fredholm integro-differential equations, but all algorithms in our work can be applied to Volterra integro-differential equations with a little modification. Integro-differential equations are important, but they are hard to solve even numerically, so the progress on how to solve them is slow. The concepts of integro-differential equations have motivated a huge size of research work in recent years, several numerical methods were used such as wavelet-Galerkin method [1], Lagrange interpolation method [2], Taylor polynomials [3] and [4], Chebyshev polynomials [5], [6] and [7], Adomian decomposition method [8] and [9], the differential transformation method [10] and [11], Legendre polynomial [12], CAS Wavelet operational matrix [13], Reduced differential transform method[14], Homotopy perturbation method [15]. In our work, we apply rational Chebyshev (RC) collocation method [16] and [17] for solving high-order linear Fredholm integro-differential equations and we will show that convergent rate of RC is more accelerate than other existing method. The organization of this paper, in Section 2, preliminaries introduced while in section 3, properties of the RC functions are presented. In Section 4, we formulated the fundamental matrix relation based on collocation Points. In Section 5, the method of solution is presented. Section 6, contains numerical illustrations and results that are compared with the exact solution and other existed methods. Finally, section 7, concludes this article with a brief summary. 


\section{Preliminaries}

Consider the $m$ th order linear Fredholm integro-differential equation with variable coefficients

$$
\sum_{k=0}^{m} P_{k}(x) y^{(k)}(x)=g(x)+\lambda \int_{0}^{a} K(x, t) y(t) d t, 0 \leq x, t \leq a<\infty,
$$

under the mixed conditions

$$
\sum_{k=0}^{m-1}\left(a_{j k} y^{(k)}(\alpha)+b_{j k} y^{(k)}(\beta)+c_{j k} y^{(k)}(\gamma)\right)=\mu_{j}, \alpha \leq \gamma \leq \beta, \quad j=0,1, \ldots, m-1
$$

where $a_{j k}, b_{j k}, c_{j k}$ and $\mu_{j}$ are suitable constants and $P_{k}(x), g(x), K(x, t)$ are function defined in interval $0 \leq x, t \leq a<\infty$. We assume that the solution of this system can be expressed in terms of a truncated RC functions as follows:

$$
y_{i}(x)=\sum_{n=0}^{N} a_{n} R_{n}(x), i=1,2, \ldots, k, 0 \leq x<\infty,
$$

where $N$ is chosen any positive integer such that $N \geq m$ and $a_{n}$ are unknown RC coefficients.

\section{Properties of the rational Chebyshev functions}

\subsection{Rational Chebyshev functions}

The well-known Chebyshev polynomials are orthogonal in the interval $[-1,1]$ with respect to the weight function $w(x)=$ $1 / \sqrt{1-x^{2}}$ and can be determined with the aid of the recurrence formulae

$$
T_{0}(x)=1, T_{1}(x)=x, T_{n+1}(x)=2 x T_{n}(x)-T_{n-1}(x) n \geq 1
$$

The RC functions are defined by

$$
R_{n}(x)=T_{n}\left(\frac{x-1}{x+1}\right)
$$

The recurrence relation is

$$
R_{0}(x)=1, R_{1}(x)=\frac{x-1}{x+1}, R_{n+1}(x)=2\left(\frac{x-1}{x+1}\right) R_{n}(x)-R_{n-1}(x), n \geq 1
$$

$\mathrm{RC}$ functions are orthogonal with respect to the weight function $w(x)=1 /((x+1) \sqrt{x})$ in the interval $[0, \infty)$, with the orthogonally property:

$$
\int_{0}^{\infty} R_{n}(x) R_{m}(x) w(x) d x=\frac{c_{m} \pi}{2} \delta_{n m}
$$

with

$$
c_{m}=\left\{\begin{array}{l}
2, m=0 \\
1, m \geq 1
\end{array}\right.
$$

where $\delta_{n m}$ is the Kronecker function. 
Since the set of RC functions is orthogonal and complete $y(x)$ defined over the interval $[0, \infty)$ can be expanded as:

$$
y(x)=\sum_{n=0}^{\infty} a_{n} R_{n}(x),
$$

where

$$
a_{i}=\frac{2}{c_{i} \pi} \int_{0}^{\infty} R_{i}(x) y(x) w(x) d x .
$$

\subsection{Fundamental matrix derivative relation of $R C$ by first scheme $(R R C)[16]$}

The derivative of the vector $R(x)=\left[R_{0}(x) R_{1}(x) \ldots R_{N}(x)\right]$ can be expressed by

$$
R^{\prime}(x)=\frac{d R(x)}{d x} \approx R(x) D^{T}
$$

where $D$ is $(N+1) \times(N+1)$ operational matrix for the derivative.

Differentiating relation (4) we get:

$$
R_{n+1}^{\prime}(x)=\frac{4}{(x+1)^{2}} R_{n}(x)+2\left(\frac{x-1}{x+1}\right) R_{n}^{\prime}(x)-R_{n-1}^{\prime}(x), n \geq 1 .
$$

The derivative of $R_{1}(x)$ is $\frac{2}{(x+1)^{2}}$ which can be expressed as follows:

$$
R_{1}^{\prime}(x)=\frac{2}{(x+1)^{2}}=\frac{3}{4} R_{0}(x)-R_{1}(x)+\frac{1}{4} R_{2}(x),
$$

Form above, the elements $d_{i j}$, of the matrix $D$ can be obtained from

$$
\left\{\begin{array}{l}
R_{0}^{\prime}(x)=0 \\
R^{\prime}{ }_{1}(x)=\frac{3}{4} R_{0}(x)-R_{1}(x)+\frac{1}{4} R_{2}(x), \\
R_{n+1}^{\prime}(x)=2\left(R_{1}(x) \cdot R_{n}(x)\right)^{\prime}-R_{n-1}^{\prime}(x), n>1,
\end{array}\right.
$$

where

$$
R_{m} R_{n}=\frac{1}{2}\left[R_{m+n}+R_{|m-n|}\right]
$$

The general form of the matrix $D$ is a lower- Heisenberg matrix. The matrix $D$ can be expressed as $D=D_{1}+D_{2}$, where $D_{1}$ is a tridiagonal matrix which is obtained from

$$
D_{1}=\text { diag. }\left(\frac{7}{4}(i-1),-(i-1), \frac{1}{4}(i-1)\right),
$$

and the $d_{i j}$ elements of matrix $D_{2}$ are obtained from $d_{21}=-1$ and

$$
d_{i j}=\left\{\begin{array}{c}
0, j \geq i-1 \\
k(i-1) c_{j}, j<i-1
\end{array}\right.
$$

where $k=(-1)^{i+j+1}, c_{1}=1$ and $c_{j}=2$ for $j \geq 2$. For $N=5$ we have 


$$
D=\left[\begin{array}{llllll}
0 & 0 & 0 & 0 & 0 & 0 \\
3 / 4 & -1 & 1 / 4 & 0 & 0 & 0 \\
-2 & 7 / 2 & -2 & 1 / 2 & 0 & 0 \\
3 & -6 & 21 / 4 & -3 & 3 / 4 & 0 \\
-4 & 8 & -8 & 7 & -4 & 1 \\
5 & -10 & 10 & -10 & 35 / 4 & -5
\end{array}\right]
$$

Consequently, the $k^{t h}$ derivative of the matrix $R(x)$ defined in (5), can be obtained as

$$
R^{(k)}(x) \approx R(x)\left(D^{T}\right)^{k}
$$

\subsection{Fundamental matrix derivative relation of $R C$ by second scheme (IRC) [17]}

It is clear that using (8) leads to a truncated last term of relation (7). Moreover in the higher derivatives the truncation is more than one term. For example using forth order derivative at $n=3$, the truncated terms will vanish in the last four terms in $R_{4}, R_{5}, R_{6}, R_{7}$. This will lead to unsatisfied approximating using RRC scheme. Therefore, we will try to improve the definition of the derivative of RC functions. It is noted that in [16] the last term is truncated to get a square matrix so the matrix product is possible.

Now, we will add a vector to (8) to keep the truncated term which will improve our approximation. This technique will be called an improved regular RC functions and denoted by IRC.

Thus, we can obtain general form as:

$$
R^{\prime}(x)=\frac{d R(x)}{d x}=R(x) D^{T}+B(x)
$$

where

$$
B(x)=\left[\begin{array}{lllll}
0 & 0 & \cdots & 0 & d_{N+1, N+2} R_{N+1}(x)
\end{array}\right]_{1 \times(N+1)}
$$

To obtain the matrix $R^{(k)}(x)$ we can use the relation (9):

$$
\begin{gathered}
R^{(0)}(x)=R(x)\left(D^{T}\right)^{0} \\
R^{(1)}(x)=R(x)\left(D^{T}\right)^{1}+B^{(0)}(x) \\
R^{(2)}(x)=R^{(1)}(x) D^{T}+B^{(1)}(x)=R(x)\left(D^{T}\right)^{2}+B^{(0)}(x) D^{T}+B^{(1)}(x) \\
R^{(3)}(x)=R^{(1)}(x)\left(D^{T}\right)^{2}+B^{(1)}(x) D^{T}+B^{(2)}(x)=R(x)\left(D^{T}\right)^{3}+B(x)\left(D^{T}\right)^{2}+B^{(1)}(x) D^{T}+B^{(2)}(x)
\end{gathered}
$$

Consequently, the $k^{\text {th }}$ derivative of the matrix $R(x)$ defined as:

$$
R^{(0)}(x)=R(x), R^{(k)}(x)=R(x)\left(D^{T}\right)^{k}+\sum_{i=0}^{k-1} B^{(i)}(x)\left(D^{T}\right)^{\mathrm{k}-\mathrm{i}-1} k \geq 1
$$


where

$$
B^{(k)}(x)=\left[\begin{array}{lllll}
0 & 0 & \cdots & 0 & d_{N+1, N+2} R_{N+1}^{(k)}(x)
\end{array}\right] .
$$

\section{Fundamental matrix relation based on collocation points}

In this section we will provide fundamental matrix relation based on collocation points of the solution of (1) with (2) by two schemes. First assume that the solution $y(x)$ of (1) can be expressed in the form (3), which is a truncated Chebyshev series in terms of RC functions. Then $y(x)$ and its derivative $y^{(j)}(x)$ can be put in the matrix forms

$$
[y(x)]=R(x) A,
$$

and

$$
\left[y^{(j)}(x)\right]=R^{(j)}(x) A, j=0,1, \ldots, m \leq N,
$$

where

$$
R^{(j)}(x)=\left[R_{0}^{(j)}(x) R_{1}^{(j)}(x) \ldots R_{N}^{(j)}(x)\right], \quad A=\left[\begin{array}{llll}
a_{0} & a_{1} & \ldots & a_{N}
\end{array}\right]^{T}
$$

Now, let us write (1) in the form

$$
\mathrm{F}(x)=g(x)+\lambda I(x)
$$

where the differential part

$$
F(x)=\sum_{k=0}^{m} P_{k}(x) y^{(k)}(x)
$$

and the Fredholm integral part

$$
I(x)=\int_{0}^{a} K(x, t) y(t) d t,
$$

Let us now form the matrix relation for Fredholm integral part (14). The kernel function $K(x, t)$ can be approximated by the truncated RC series as follows

$$
K(x, t)=\sum_{l=0}^{N} \sum_{s=0}^{N} k_{l s} R_{l}(x) R_{s}(t)
$$

where

$$
k_{l s}=\frac{4}{c_{l} c_{s} \pi^{2}} \int_{0}^{\infty} \int_{0}^{\infty} R_{l}(x) R_{S}(t) K(x, t) w(x) w(t) d x d t .
$$

Then the matrix representation of $K(x, t)$ can be given by

$$
[K(x, t)]=R(x) K R^{T}(t)
$$

where

$$
K=\left[\begin{array}{llll}
k_{00} & k_{01} & \ldots & k_{0 N} \\
k_{10} & k_{11} & \ldots & k_{1 N} \\
\vdots & \vdots & \ddots & \vdots \\
k_{N 0} & k_{N 1} & \ldots & k_{N N}
\end{array}\right]
$$


The matrix representation of $y(t)$ can be given by

$$
[y(t)]=R(t) A
$$

Substituting (15), (16) into (14), we get

$$
[I(x)]=\int_{0}^{a} R(x) K R^{T}(t) R(t) A d t=R(x) K M A,
$$

where

$$
M=\int_{0}^{a} R^{T}(t) R(t) d t
$$

\subsection{Fundamental matrix relation by first scheme (RRC)}

From relations (8) and (11), then (13), takes the form

$$
F(x)=\sum_{k=0}^{m} P_{k}(x) R(x)\left(D^{T}\right)^{k} A
$$

Substituting (17) and (18) into (12), we get

$$
\sum_{k=0}^{m} P_{k}(x) R(x)\left(D^{T}\right)^{k} A=g(x)+\lambda R(x) K M A
$$

Now, let us define the collocation points as

$$
s=0,1, \ldots, N
$$

Upon substitu $x_{s}=\frac{c}{N} s$, ting points (20) into (19) we obtain

$$
\sum_{k=0}^{m} P_{k}\left(x_{s}\right) R\left(x_{s}\right)\left(D^{T}\right)^{k} A=g\left(x_{s}\right)+\lambda R\left(x_{s}\right) K M A
$$

The obtained system (21) can be written farther in the matrix form

$$
\sum_{k=0}^{m} \mathrm{P}_{\mathrm{k}} \mathrm{R}\left(D^{T}\right)^{k} A=\mathrm{G}+\lambda \mathrm{R} K M A
$$

where

$$
\begin{gathered}
\mathrm{P}_{k}=\left[\begin{array}{cccc}
P_{k}\left(x_{0}\right) & 0 & \ldots & 0 \\
0 & P_{k}\left(x_{1}\right) & \ldots & 0 \\
0 & 0 & \ddots & \vdots \\
0 & 0 & \ldots & P_{k}\left(x_{N}\right)
\end{array}\right], \mathrm{G}=\left[\begin{array}{c}
g\left(x_{0}\right) \\
g\left(x_{1}\right) \\
\vdots \\
g\left(x_{N}\right)
\end{array}\right] \\
\mathrm{R}=\left[\begin{array}{c}
R\left(x_{0}\right) \\
R\left(x_{1}\right) \\
\vdots \\
R\left(x_{N}\right)
\end{array}\right]=\left[\begin{array}{cccc}
R_{0}\left(x_{0}\right) & R_{1}\left(x_{0}\right) & \ldots & R_{N}\left(x_{0}\right) \\
R_{0}\left(x_{1}\right) & R_{1}\left(x_{1}\right) & \ldots & R_{N}\left(x_{1}\right) \\
\vdots & \vdots & \ddots & \vdots \\
R_{0}\left(x_{N}\right) & R_{1}\left(x_{N}\right) & \ldots & R_{N}\left(x_{N}\right)
\end{array}\right] .
\end{gathered}
$$


Then the fundamental matrix for solving of Fredholm integro-differential equation obtains as:

$$
\left(\sum_{k=0}^{m} \mathrm{P}_{\mathrm{k}} \mathrm{R}\left(D^{T}\right)^{k}-\lambda \mathrm{R} K M\right) A=\mathrm{G}
$$

Similarly, we form the matrix representations of the mixed conditions.

Substituting the matrices $y^{(k)}(\alpha), y^{(k)}(\beta)$ and $y^{(k)}(\gamma)$ which depends on the RC coefficients matrix $A$ into (2) and simplifying the result we obtain

$$
\sum_{k=0}^{m-1}\left\{a_{j k} R(\alpha)+b_{j k} R(\beta)+c_{j k} R(\gamma)\right\}\left(D^{T}\right)^{k} A=\mu_{j}
$$

\subsection{Fundamental matrix relation by second scheme (IRC)}

Similarly, the second scheme IRC gives us derivative RC functions of $k^{\text {th }}$ order as:

$$
R^{(0)}(x)=R(x), R^{(k)}(x)=R(x)\left(D^{T}\right)^{k}+\sum_{i=0}^{k-1} B^{(i)}(x)\left(D^{T}\right)^{k-i-1}, k \geq 1 .
$$

From relations (10) and (11), then (13), takes the form

$$
F(x)=\sum_{k=0}^{m} \mathrm{P}_{k}(x)\left\{\mathrm{R}(\mathrm{x})\left(D^{T}\right)^{k}+\sum_{i=0}^{k-1} \mathrm{~B}^{(i)}(\mathrm{x})\left(D^{T}\right)^{\mathrm{k}-\mathrm{i}-1}\right\} A,
$$

Substituting (17) and (25) into (12), we get

$$
\sum_{k=0}^{m} P_{k}(x)\left\{\mathrm{R}(\mathrm{x})\left(D^{T}\right)^{k}+\sum_{i=0}^{k-1} \mathrm{~B}^{(i)}(\mathrm{x})\left(D^{T}\right)^{\mathrm{k}-\mathrm{i}-1}\right\} A=g(x)+\lambda R(x) K M A .
$$

Upon substituting points (20) into (26) we obtain

$$
\sum_{k=0}^{m} P_{k}\left(x_{s}\right)\left\{\mathrm{R}\left(\mathrm{x}_{\mathrm{s}}\right)\left(D^{T}\right)^{k}+\sum_{i=0}^{k-1} \mathrm{~B}^{(i)}\left(\mathrm{x}_{\mathrm{s}}\right)\left(D^{T}\right)^{\mathrm{k}-\mathrm{i}-1}\right\} A=g\left(x_{s}\right)+\lambda R\left(x_{s}\right) K M A
$$

The obtained system (27) can be written farther in the matrix form

$$
\sum_{k=0}^{m} \mathrm{P}_{\mathrm{k}}\left\{\mathrm{R}\left(D^{T}\right)^{k}+\sum_{i=0}^{k-1} \mathrm{~B}^{(i)}\left(D^{T}\right)^{\mathrm{k}-\mathrm{i}-1}\right\} A=\mathrm{G}+\lambda \mathrm{R} K M A
$$

where

$$
\mathrm{P}_{k}=\left[\begin{array}{cccc}
P_{k}\left(x_{0}\right) & 0 & \ldots & 0 \\
0 & P_{k}\left(x_{1}\right) & \ldots & 0 \\
0 & 0 & \ddots & \vdots \\
0 & 0 & \ldots & P_{k}\left(x_{N)}\right.
\end{array}\right], \mathrm{G}=\left[\begin{array}{c}
g\left(x_{0}\right) \\
g\left(x_{1}\right) \\
\vdots \\
g\left(x_{N}\right)
\end{array}\right]
$$




$$
\begin{gathered}
\mathrm{R}=\left[\begin{array}{c}
R\left(x_{0}\right) \\
R\left(x_{1}\right) \\
\vdots \\
R\left(x_{N}\right)
\end{array}\right]=\left[\begin{array}{cccc}
R_{0}\left(x_{0}\right) & R_{1}\left(x_{0}\right) & \ldots & R_{N}\left(x_{0}\right) \\
R_{0}\left(x_{1}\right) & R_{1}\left(x_{1}\right) & \ldots & R_{N}\left(x_{1}\right) \\
\vdots & \vdots & \ddots & \vdots \\
R_{0}\left(x_{N}\right) & R_{1}\left(x_{N}\right) & \ldots & R_{N}\left(x_{N}\right)
\end{array}\right], \\
\mathrm{B}=\left[\begin{array}{c}
B\left(x_{0}\right) \\
B\left(x_{1}\right) \\
\vdots \\
B\left(x_{N}\right)
\end{array}\right]=\left[\begin{array}{cccc}
B_{0}\left(x_{0}\right) & B_{1}\left(x_{0}\right) & \ldots & B_{N}\left(x_{0}\right) \\
B_{0}\left(x_{1}\right) & B_{1}\left(x_{1}\right) & \ldots & B_{N}\left(x_{1}\right) \\
\vdots & \vdots & \ddots & \vdots \\
B_{0}\left(x_{N}\right) & B_{1}\left(x_{N}\right) & \ldots & B_{N}\left(x_{N}\right)
\end{array}\right] .
\end{gathered}
$$

Then the fundamental matrix for solving of Fredholm integro-differential equation takes the form:

$$
\left(\sum_{k=0}^{m} \mathrm{P}_{\mathrm{k}}\left\{\mathrm{R}\left(D^{T}\right)^{k}+\sum_{i=0}^{k-1} \mathrm{~B}^{(i)}\left(D^{T}\right)^{\mathrm{k}-\mathrm{i}-1}\right\}-\lambda \mathrm{R} K M\right) A=\mathrm{G}
$$

Similarly, we form the matrix representations of the mixed conditions.

Substituting the matrix $y^{(k)}(\alpha), y^{(k)}(\beta)$ and $y^{(k)}(\gamma)$ which depends on the RC coefficients matrix $A$ into the (5.2) and simplifying the result we obtain

$$
\begin{aligned}
& \sum_{k=0}^{m-1}\left\{a_{j k}\left\{\mathrm{R}(\alpha)\left(D^{T}\right)^{k}+\sum_{i=0}^{k-1} \mathrm{~B}^{(i)}(\alpha)\left(D^{T}\right)^{\mathrm{k}-\mathrm{i}-1}\right\}+b_{j k}\left\{\mathrm{R}(\beta)\left(D^{T}\right)^{k}+\right.\right. \\
& \left.\left.\sum_{i=0}^{k-1} \mathrm{~B}^{(i)}(\beta)\left(D^{T}\right)^{\mathrm{k}-\mathrm{i}-1}\right\}+c_{j k}\left\{\mathrm{R}(\gamma)\left(D^{T}\right)^{k}+\sum_{i=0}^{k-1} \mathrm{~B}^{(i)}(\gamma)\left(D^{T}\right)^{\mathrm{k}-\mathrm{i}-1}\right\}\right\} A=\mu_{j} .
\end{aligned}
$$

\section{Method of solution}

The fundamental matrix equations (23) and (29) for (1) correspond to a system of $(N+1)$ algebraic equations for the $(N+1)$ unknown coefficients $a_{0}, a_{1}, \ldots, a_{N}$. One writes equations (23) and (29) in short form as:

$$
W A=\mathbf{G} \quad \text { or } \quad[W ; \mathbf{G}]
$$

We can obtain the matrix form for the mixed conditions (2), by means of equations (24) and (30) briefly as

$$
U_{i} A=\left[\lambda_{i}\right]
$$

so that $W$ and $U_{i}$ for first scheme defined by:

$$
W=\left[w_{p q}\right]=\sum_{k=0}^{m} \mathrm{P}_{\mathrm{k}} \mathrm{R}\left(D^{T}\right)^{k}-\lambda \mathrm{R} K M, p, q=0,1, \ldots, N
$$

and

$$
U_{i}=\left[\begin{array}{llll}
u_{i 0} & u_{i 1} & \ldots & u_{i N}
\end{array}\right]=\sum_{k=0}^{m-1}\left\{a_{j k} R(\alpha)+b_{j k} R(\beta)+c_{j k} R(\gamma)\right\}\left(D^{T}\right)^{k}
$$

while, the definition of $W$ and $U_{i}$ for second scheme obtained as:

$$
W=\left[w_{p q}\right]=\sum_{k=0}^{m} \mathrm{P}_{k}\left\{\mathrm{R}\left(D^{T}\right)^{k}+\sum_{i=0}^{k-1} \mathrm{~B}^{(i)}\left(D^{T}\right)^{\mathrm{k}-\mathrm{i}-1}\right\}
$$


and

$$
\begin{aligned}
& U_{i}=\left[\begin{array}{llll}
u_{i 0} & u_{i 1} & \ldots & u_{i N}
\end{array}\right]=\sum_{k=0}^{m-1} a_{j k}\left\{R(\alpha)\left(D^{T}\right)^{k}+\sum_{i=0}^{k-1} B^{(i)}(\alpha)\left(D^{T}\right)^{\mathrm{k}-\mathrm{i}-1}\right\}+ \\
& b_{j k}\left\{R(\beta)\left(D^{T}\right)^{k}+\sum_{i=0}^{k-1} B^{(i)}(\beta)\left(D^{T}\right)^{\mathrm{k}-\mathrm{i}-1}\right\}+c_{j k}\left\{R(\gamma)\left(D^{T}\right)^{k}+\sum_{i=0}^{k-1} B^{(i)}(\gamma)\left(D^{T}\right)^{\mathrm{k}-\mathrm{i}-1}\right\}
\end{aligned}
$$

Now, the solution of (1) under the conditions (2), can then be obtained by replacing the rows of matrices (32) by the last $m$ rows of the matrix (31), we get the required augmented matrix

$$
[\tilde{W} ; \tilde{\mathrm{G}}]=\left[\begin{array}{llllll}
w_{00} & w_{01} & \ldots & w_{0 N} & ; & g\left(x_{0}\right) \\
w_{10} & w_{11} & \ldots & w_{1 N} & ; & g\left(x_{1}\right) \\
\ldots & \ldots & \ldots & \ldots & ; & \ldots \\
w_{N-m, 0} & w_{N-m, 1} & \ldots & w_{N-m, N} & g\left(x_{N-m}\right) \\
u_{00} & u_{01} & \ldots & u_{0 N} & ; & \lambda_{0} \\
u_{10} & u_{11} & \ldots & u_{1 N} & ; & \lambda_{1} \\
\ldots & \ldots & \ldots & \ldots & ; & \ldots \\
u_{m-1,0} & u_{m-1,1} & \ldots & u_{m-1, N} & ; & \lambda_{m-1}
\end{array}\right] .
$$

If $\operatorname{rank} \tilde{W}=\operatorname{rank}[\tilde{W} ; \tilde{\mathrm{G}}]=N+1$, then we can write the matrix equation (31) as:

$$
A=(\tilde{W})^{-1} \tilde{\mathrm{G}}
$$

and therefore the coefficients $a_{n} ; n=0,1, \ldots, N$ are uniquely determined by (33).

\section{Numerical examples}

In this section, numerical examples are given to illustrate the applicability, accuracy and effectiveness of the proposed techniques. All examples are performed on the computer using a program written in MATHEMATICA 7.0. The obtained numerical results are presented as shown in the illustrative Tables. The absolute errors, in tables, are given by the values of $e(x)=\left|y(x)-y_{N}(x)\right|$ evaluated at selected points.

Example 1. Let us first consider the linear Fredholm integro-differential equation

$$
y^{\prime \prime}(x)-\frac{2}{(1+x)^{2}} y(x)=\frac{\ln [11]}{1+x}-\int_{0}^{10} \frac{1}{1+x} y(t) d t, \quad x \in[0,10],
$$

with $y(0)=1, y(1)=\frac{1}{2}$. For this example we have,

$$
m=2, P_{0}(x)=\frac{-2}{(x+1)^{2}}, P_{1}(x)=0, P_{2}(x)=1, g(x)=\frac{\ln [11]}{x+1}, K(x, t)=\frac{1}{x+1} .
$$

Then for $N=2$, the collocation points are

$$
x_{0}=0, x_{1}=5, x_{2}=10
$$

Where $\mathrm{P}_{0}, \mathrm{P}_{1}, \mathrm{P}_{2}, K$, Mare matrices of order $(3 \times 3)$ defined by

$$
\mathrm{P}_{0}=\left[\begin{array}{lll}
-2 & 0 & 0 \\
0 & -1 / 18 & 0 \\
0 & 0 & -2 / 121
\end{array}\right], \mathrm{P}_{1}=\left[\begin{array}{lll}
0 & 0 & 0 \\
0 & 0 & 0 \\
0 & 0 & 0
\end{array}\right], \mathrm{P}_{2}=\left[\begin{array}{lll}
1 & 0 & 0 \\
0 & 1 & 0 \\
0 & 0 & 1
\end{array}\right]
$$




$$
K=\left[\begin{array}{lrl}
1 / 2 & 0 & 0 \\
-1 / 2 & 0 & 0 \\
0 & 0 & 0
\end{array}\right], M=\left[\begin{array}{lll}
10 & -2(-5+\ln [11]) & \frac{190}{11}-8 \ln [11] \\
-2(-5+\ln [11]) & \frac{150}{11}-4 \ln [11] & \frac{2890}{121}-10 \ln [11] \\
\frac{190}{11}-8 \ln [11] & \frac{2890}{121}-10 \ln [11] & \frac{162010}{3993}-16 \ln [11]
\end{array}\right],
$$

The augmented matrix forms of the conditions for $N=2$ are

$$
\left[\begin{array}{lllll}
1 & -1 & 1 & ; & 1
\end{array}\right],[10-1 ; 1 / 2],
$$

The fundamental matrix equation by IRC scheme (29) of problem is

$$
\begin{gathered}
\left(\mathrm{P}_{0} \mathrm{R}+\mathrm{P}_{1}\left(\mathrm{R} D^{T}+\mathrm{B}\right)+\mathrm{P}_{2}\left(\mathrm{R}\left(D^{T}\right)^{2}+\mathrm{B} D^{T}+\mathrm{B}^{\prime}\right)-\lambda \mathrm{RKM}\right) A=\mathrm{G} \\
\mathrm{R}=\left[\begin{array}{ccc}
1 & -1 & 1 \\
1 & \frac{2}{3} & -\frac{1}{9} \\
1 & \frac{9}{11} & \frac{41}{121}
\end{array}\right], \quad D^{T}=\left[\begin{array}{lll}
0 & \frac{3}{4} & -2 \\
0 & -1 & \frac{7}{2} \\
0 & \frac{1}{4} & -2
\end{array}\right], \quad\left(D^{T}\right)^{2}=\left[\begin{array}{ccc}
0 & -\frac{5}{4} & \frac{53}{8} \\
0 & \frac{15}{8} & -\frac{21}{2} \\
0 & -\frac{3}{4} & \frac{39}{8}
\end{array}\right], \\
\mathrm{B}=\left[\begin{array}{lll}
0 & 0 & -\frac{1}{2} \\
0 & 0 & -\frac{11}{27} \\
0 & 0 & -\frac{351}{2662}
\end{array}\right], \quad \mathrm{B}^{\prime}=\left[\begin{array}{lll}
0 & 0 & 9 \\
0 & 0 & \frac{7}{108} \\
0 & 0 & \frac{609}{14641}
\end{array}\right] .
\end{gathered}
$$

Then, we obtain the augmented matrix (33) as

$$
[\tilde{W} ; \tilde{\mathrm{G}}]=\left[\begin{array}{cccc}
8-2-2(-5+\ln [11] & \frac{520}{11}-8 \ln [11] & ; \ln [11] \\
1 & -1 & 1 & ; 1 \\
1 & 0 & -1 & ; 1 / 2
\end{array}\right]
$$

we then obtain the solution for $\mathrm{WA}=\boldsymbol{G}$

$$
A=[1 / 2-1 / 20]
$$

Therefore, we find the solution

$$
y(x)=\sum_{n=0}^{2} a_{n} R_{n}(x)
$$

to be in the form

$$
y(x)=\frac{1}{2} R_{0}(x)-\frac{1}{2} R_{1}(x),
$$

or in the form

$$
y(x)=\frac{1}{x+1},
$$

which is exact solution of this problem.

The fundamental matrix equation (23) of problem by RRC scheme is

$$
\left(\mathrm{P}_{0} \mathrm{R}+\mathrm{P}_{1} \mathrm{R} D^{T}+\mathrm{P}_{2} \mathrm{R}\left(D^{T}\right)^{2}-\lambda \mathrm{R} K M\right) A=\mathrm{G}
$$

for $N=2$ we get badly approximate but for $N=3$ we will get the exact solution of this problem. 
Example 2. Let us first consider the integro-differential equation [10], [13]

$$
y^{\prime}(x)=1-\frac{1}{3} x+\int_{0}^{1} x t y(t) d t, \quad x \in[0,1]
$$

with $y(0)=0$. The numerical solutions obtained from the IRC scheme and RRC scheme for $N=4$. In table 1 the errors given by the two proposed schemes compared with errors calculated by CAS Wavelet method [13] and differential transformation method [10] in the interval[0,1]. It is noted from table 1 that the present method by two schemes is better than the differential transform method in [10]. On the other hand, the results obtained by CAS Wavelet method [13] are close to the results obtained by the present method.

Table 1: Comparison between absolute error obtained by present method and other existed methods for $y(x)$ of Exam. 2 .

\begin{tabular}{|c|l|c|c|c|}
\hline $\boldsymbol{X}$ & \multicolumn{1}{|c|}{$\begin{array}{c}\text { CAS } \\
\text { Wavelet[13] }\end{array}$} & $\begin{array}{c}\text { Differential } \\
\text { transformation } \\
\text { method[10] }\end{array}$ & $\begin{array}{c}\text { RRC } \\
\boldsymbol{N}=\mathbf{4}\end{array}$ & $\begin{array}{l}\text { IRC } \\
\boldsymbol{N}=\mathbf{4}\end{array}$ \\
\hline 0.1 & $2.17942375 \mathrm{e}-004$ & $1.66666667 \mathrm{e}-003$ & $4.44627459 \mathrm{e}-004$ & $7.48179732 \mathrm{e}-004$ \\
\hline 0.2 & $6.38548213 \mathrm{e}-004$ & $6.09388620 \mathrm{e}-003$ & $5.89430306 \mathrm{e}-004$ & $1.63253385 \mathrm{e}-003$ \\
\hline 0.3 & $7.91370487 \mathrm{e}-004$ & $1.32017875 \mathrm{e}-002$ & $2.40171141 \mathrm{e}-003$ & $3.51161586 \mathrm{e}-003$ \\
\hline 0.4 & $2.15586005 \mathrm{e}-002$ & $2.29140636 \mathrm{e}-002$ & $6.73535353 \mathrm{e}-003$ & $7.27152372 \mathrm{e}-003$ \\
\hline 0.5 & $4.99358429 \mathrm{e}-003$ & $3.51578404 \mathrm{e}-002$ & $1.40138093 \mathrm{e}-002$ & $1.36200176 \mathrm{e}-002$ \\
\hline 0.6 & $2.21728815 \mathrm{e}-002$ & $6.69648304 \mathrm{e}-002$ & $2.44620175 \mathrm{e}-002$ & $2.30527344 \mathrm{e}-002$ \\
\hline 0.7 & $1.05645449 \mathrm{e}-004$ & $7.12430514 \mathrm{e}-002$ & $3.81931054 \mathrm{e}-002$ & $3.58782889 \mathrm{e}-002$ \\
\hline 0.8 & $1.43233681 \mathrm{e}-003$ & $8.63983845 \mathrm{e}-002$ & $5.52463654 \mathrm{e}-002$ & $5.22579230 \mathrm{e}-002$ \\
\hline 0.9 & $2.07747461 \mathrm{e}-002$ & $1.08103910 \mathrm{e}-001$ & $7.56088331 \mathrm{e}-002$ & $7.22435281 \mathrm{e}-002$ \\
\hline
\end{tabular}

Example 3. Consider the following linear Fredholm integro -differential equation [10]

$$
y^{\prime}(x)=x e^{x}+e^{x}-x \int_{0}^{1} x y(t) d t \quad x \in[0,1]
$$

with $y(0)=0$.

The numerical solutions obtained by the IRC scheme for $N=4$. In table 2 comparing the error obtained with CAS wavelet method [13] and differential transformation method [10].

Table 2: Comparison between absolute error obtained by present method and other existed methods for $y(x)$ of Exam. 3 .

\begin{tabular}{|c|c|c|c|}
\hline $\boldsymbol{X}$ & CAS Wavelet[13] & DTM[10] & IRC $\boldsymbol{N}=\mathbf{4}$ \\
\hline 0.1 & $1.34917637 \mathrm{e}-003$ & $1.00118319 \mathrm{e}-002$ & $2.33899689 \mathrm{e}-002$ \\
\hline 0.2 & $1.15960044 \mathrm{e}-003$ & $2.78651355 \mathrm{e}-002$ & $4.32236340 \mathrm{e}-002$ \\
\hline 0.3 & $5.67152531 \mathrm{e}-003$ & $5.08730892 \mathrm{e}-002$ & $4.19865348 \mathrm{e}-002$ \\
\hline 0.4 & $5.93105645 \mathrm{e}-002$ & $7.55356316 \mathrm{e}-002$ & $2.88393798 \mathrm{e}-002$ \\
\hline 0.5 & $1.32330751 \mathrm{e}-002$ & $9.71888592 \mathrm{e}-002$ & $1.16259014 \mathrm{e}-002$ \\
\hline 0.6 & $4.39287720 \mathrm{e}-002$ & $1.09551714 \mathrm{e}-001$ & $8.45940052 \mathrm{e}-003$ \\
\hline 0.7 & $1.41201624 \mathrm{e}-002$ & $1.04133232 \mathrm{e}-001$ & $3.62299942 \mathrm{e}-002$ \\
\hline 0.8 & $1.34514117 \mathrm{e}-002$ & $6.94512700 \mathrm{e}-002$ & $8.08146109 \mathrm{e}-002$ \\
\hline 0.9 & $1.32045209 \mathrm{e}-002$ & $1.00034260 \mathrm{e}-002$ & $1.54221606 \mathrm{e}-001$ \\
\hline
\end{tabular}


Example 4. Consider the third order linear intgro-differential equation

$$
y^{\prime \prime \prime}(x)+x y^{\prime}(x)+x y(x)=e^{-x}-e+1+e \int_{0}^{1} y(t) d t,
$$

with $y(0)=1, y^{\prime}(0)=-1, y^{\prime \prime}(0)=1$.

The exact solution of this problem is $y(x)=e^{-x}$. The solution obtained by IRC scheme. In table 3 the errors calculated with different $N$, clearly indicates that when we increase the truncation limit $N$, we have good accuracy.

Table 3: Comparison between absolute error functions obtained by IRC scheme for $y(x)$ of Example 4 for $N=8,10,12$ and 16.

\begin{tabular}{|c|c|c|c|c|}
\hline$X$ & $e_{N=8}$ & $e_{N=10}$ & $e_{N=12}$ & $e_{N=16}$ \\
\hline 0.1 & $2.29514 \mathrm{e}-007$ & $8.41043 \mathrm{e}-008$ & $2.45139 \mathrm{e}-008$ & $2.21017 \mathrm{e}-008$ \\
\hline 0.2 & $1.54099 \mathrm{e}-006$ & $5.06277 \mathrm{e}-007$ & $1.35645 \mathrm{e}-007$ & $1.83008 \mathrm{e}-008$ \\
\hline 0.3 & $4.08800 \mathrm{e}-006$ & $1.29587 \mathrm{e}-006$ & $3.39746 \mathrm{e}-007$ & $1.00211 \mathrm{e}-008$ \\
\hline 0.4 & $7.89477 \mathrm{e}-006$ & $2.46579 \mathrm{e}-006$ & $6.40228 \mathrm{e}-007$ & $2.9693 \mathrm{e}-009$ \\
\hline 0.5 & $1.30008 \mathrm{e}-005$ & $4.02634 \mathrm{e}-006$ & $1.03977 \mathrm{e}-006$ & $1.77792 \mathrm{e}-008$ \\
\hline 0.6 & $1.94259 \mathrm{e}-005$ & $5.98439 \mathrm{e}-006$ & $1.54011 \mathrm{e}-006$ & $3.25369 \mathrm{e}-008$ \\
\hline 0.7 & $2.71767 \mathrm{e}-005$ & $8.34151 \mathrm{e}-006$ & $2.14169 \mathrm{e}-006$ & $4.64512 \mathrm{e}-008$ \\
\hline 0.8 & $3.62373 \mathrm{e}-005$ & $1.10928 \mathrm{e}-005$ & $2.84334 \mathrm{e}-006$ & $5.93424 \mathrm{e}-008$ \\
\hline 0.9 & $4.65865 \mathrm{e}-005$ & $1.42107 \mathrm{e}-005$ & $3.64141 \mathrm{e}-006$ & $7.13077 \mathrm{e}-008$ \\
\hline 1.0 & $5.82724 \mathrm{e}-005$ & $1.75925 \mathrm{e}-005$ & $4.52514 \mathrm{e}-006$ & $8.25347 \mathrm{e}-008$ \\
\hline
\end{tabular}

Example 5.Consider the following linear Fredholm integro -differential equation

$$
x y^{\prime}(x)-y(x)=\frac{3+\operatorname{Ln}[16]}{1+x}-2 \int_{0}^{3} \frac{t}{x+x t+t+1} y(t) d t \quad x \in[0,3]
$$

With $y(0)=0$. The exact solution is $y(x)=x$.

The errors given for various $N$ in interval $[0,3]$ using IRC scheme, see table 4 clearly indicates that when we increase the $N$, we have less error.

Table 4: Comparison between absolute error obtained by IRC scheme for $y(x)$ Example 5. for $N=4,7$ and 10 .

\begin{tabular}{|c|c|c|c|}
\hline$X$ & $e_{N=4}$ & $e_{N=7}$ & $e_{N=10}$ \\
\hline 0.3 & $9.84227 \mathrm{e}-002$ & $7.99667 \mathrm{e}-003$ & $3.10157 \mathrm{e}-003$ \\
\hline 0.6 & $5.5861 \mathrm{e}-003$ & $2.51914 \mathrm{e}-003$ & $9.65748 \mathrm{e}-005$ \\
\hline 0.9 & $1.57062 \mathrm{e}-002$ & $2.35197 \mathrm{e}-004$ & $3.30361 \mathrm{e}-004$ \\
\hline 1.2 & $5.07752 \mathrm{e}-003$ & $8.6475 \mathrm{e}-004$ & $4.2555 \mathrm{e}-004$ \\
\hline 1.5 & $7.13778 \mathrm{e}-004$ & $1.04015 \mathrm{e}-003$ & $5.34414 \mathrm{e}-004$ \\
\hline 1.8 & $3.9221 \mathrm{e}-003$ & $1.20405 \mathrm{e}-003$ & $6.40535 \mathrm{e}-004$ \\
\hline 2.1 & $9.06935 \mathrm{e}-003$ & $1.45553 \mathrm{e}-003$ & $7.47712 \mathrm{e}-004$ \\
\hline 2.4 & $1.02842 \mathrm{e}-002$ & $1.62365 \mathrm{e}-003$ & $8.54097 \mathrm{e}-004$ \\
\hline 2.7 & $3.17202 \mathrm{e}-003$ & $1.82889 \mathrm{e}-003$ & $9.61814 \mathrm{e}-004$ \\
\hline 3.0 & $150938 \mathrm{e}-002$ & $2.61861 \mathrm{e}-003$ & $1.05492 \mathrm{e}-004$ \\
\hline
\end{tabular}


Example 6.Consider the following linear Fredholm integro -differential equation

$$
y^{\prime}(x)+y(x)=\operatorname{sech}(x)(1-\tanh (x))-2 \arctan \left(\frac{1-e}{1+e}\right)-\int_{0}^{1} y(t) d t,
$$

with $y(0)=1$, and the exact solution is $y(x)=\sec h(x)$.

We obtain the approximate solution by RC collocation methods of the problem for $N=8$. In table 5 the numerical results obtained by RRC and IRC schemes for $N=8$, are compared with the exact solution of this problem. Also the absolute errors for $N=8$ by the two schemes is given. The values of the solution $y(x), x \in[0,1]$ plotted with its error in figure 1 . From figure 1 one can see that the numerical results given by IRC scheme is better than RRC scheme.

Table 5: Comparison between RRC scheme and IRC scheme for $y(x)$ of Example 5.6 for $N=8$

\begin{tabular}{|l|c|c|c|c|c|}
\hline \multirow{2}{*}{$x_{i}$} & \multirow{2}{*}{ Exact solution } & \multicolumn{2}{|c|}{ RRC scheme } & \multicolumn{2}{c|}{ IRC scheme } \\
\cline { 3 - 6 } & & $N=8$ & $e R R C_{N=8}$ & $N=8$ & $e I R C_{N=8}$ \\
\hline 0.1 & 0.9950207489 & 0.9950207489 & $7.29723 \mathrm{e}-006$ & 0.9949980631 & $2.26858 \mathrm{e}-005$ \\
\hline 0.2 & 0.9803279976 & 0.9803279976 & $7.33906 \mathrm{e}-005$ & 0.9803094963 & $1.85014 \mathrm{e}-005$ \\
\hline 0.3 & 0.9566279119 & 0.9566279119 & $1.75493 \mathrm{e}-005$ & 0.9566124111 & $1.55007 \mathrm{e}-005$ \\
\hline 0.4 & 0.9250074519 & 0.9250935827 & $8.61309 \mathrm{e}-005$ & 0.9249942557 & $1.31962 \mathrm{e}-005$ \\
\hline 0.5 & 0.8868188839 & 0.8868188839 & $1.17001 \mathrm{e}-003$ & 0.8868188839 & $1.07932 \mathrm{e}-005$ \\
\hline 0.6 & 0.8435506876 & 0.8436099949 & $5.93073 \mathrm{e}-003$ & 0.8435506876 & $8.82769 \mathrm{e}-006$ \\
\hline 0.7 & 0.7967054599 & 0.7966634642 & $4.19957 \mathrm{e}-003$ & 0.7967054599 & $6.90692 \mathrm{e}-006$ \\
\hline 0.8 & 0.7476999182 & 0.7475634972 & $1.36421 \mathrm{e}-003$ & 0.7476999182 & $5.25349 \mathrm{e}-006$ \\
\hline 0.9 & 0.6977946411 & 0.6976034887 & $1.91152 \mathrm{e}-003$ & 0.6977946411 & $3.85176 \mathrm{e}-006$ \\
\hline 1.0 & 0.6480542737 & 0.6478641197 & $1.90154 \mathrm{e}-003$ & 0.6480542737 & $1.73767 \mathrm{e}-006$ \\
\hline
\end{tabular}



Fig. 1: Error function of $y(x)$ for Ex. 6 for $N=8$

\section{Conclusions}

The rational Chebyshev (RC) collocation method is proposed to find approximate solution and also, analytical solution of Fredholm integro-differential equation with variable coefficients under the mixed conditions. The proposed method converts the integral equation and its conditions to matrix equations, by means of collocation points. Moreover, the method shows to best advantage when the functions $K(x, t)$ can be expanded to the $\mathrm{RC}$ series which converges rapidly. Illustrative examples are included to demonstrate the validity and applicability of this technique, and performed on the computer using 
MATHMATICA 7.0. In addition, an interesting feature of this method is to find the analytical solution if the equation has an exact solution of rational form. Suggested approximations make this method very attractive and contributed to the good agreement between approximate and exact values in the numerical example. As a result, the power of the employed method is confirmed.

\section{References}

[1] A. Avudainayagam, C. Vani, Wavelet-Galerkin method for integro-differential equations, Appl. Numer. Math. 32 (2000) $247-254$.

[2] M. T. Rashed, Lagrange interpolation to compute the numerical solutions of differential, integral and integro differential equations, Appl. Math. Comput., 151(2004) 869- 878.

[3] M. Sezer, M. Gülsu, A new polynomial approach for solving difference and Fredholm integro-difference equations with mixed argument, Appl. Math. Comput. 171(2005) 332-344.

[4] A. Karamete, M. Sezer, A. Taylor, A collocation method for the solution of linear integro-differential equations, Intern. J. Computer Math., 79(9) (2002) 987-1000.

[5] M. Dehghan, A. Saadatmandi, Chebyshev finite difference method for Fredholm integro-differential equation, Inter. J. Comp. Math. , 85(1) (2008)123-130.

[6] M. Sezer, M. Gülsu, Y. Öztürk, A new collocation method for solution of mixed linear integro-differential-difference equations, Appl. Math. Comput. 216(2010) 2183-2198.

[7] M. Sezer, M. Gülsu, G. Yüksel, A Chebyshev polynomial approach for high-order linear Fredholm-Volterra integro-differential equations, Gazi University J. Sci., 25(2)(2012)393-401.

[8] A. R. Vahidi, E. Babolian, Gh. Cordshooli , Z. Azimzadeh , Numerical Solution of Fredholm Integro-Differential Equation by Adomian's Decomposition Method, Int. J. Math. Anal., 3(36) (2009)1769 - 1773.

[9] S. M. El-Sayed, M. R. Abdel-Aziz, A comparison of Adomian's decomposition method and Wavelet-Galerkin method for solving integro-differential equations, Appl. Math. Comput. 136(2003)151-159.

[10] P. Darania, A. Ebadian, A method for the numerical solution of the integro-differential equations, Appl. Math. Comput. 188 (2007) $657-668$.

[11] S. H. Behiry, S. I. Mohamed, Solving high-order nonlinear Volterra-Fredholm integro-differential equations by differential transform method, Natural Science, 4(8) (2012)581-587.

[12] S. Yalcinbas, M. Sezer and Hüseyin Hilmi Sorkun, Legendre polynomial solutions of high-order linear Fredholm integrodifferential equations, Appl. Math. Comput. 210(2009) 334-349.

[13] H. Danfu, Sh. Xufeng, Numerical solution of integro-differential equations by applying CAS Wavelet operational matrix of integration, Appl. Math. Comput. 194(2007) 460-466.

[14] K. Yildirim, A Solution Method For Solving Systems Of Nonlinear Pdes, World Appl. Science J, 18 (11) (2012) $1527-1532$.

[15] K. Yildirim, M. Bayram, Approximates Method for Solving an Elasticity Problem of Settled of the Elastic Ground with Variable Coefficients, Appl. Math. \& Info. Science, 7(4) (2013) 1351-1357.

[16] M. Sezer, M. Gulsu B. Tanay, Rational Chebyshev collocation method for solving higher-order linear ordinary differential equations, Wiley Online Library, DOI 10.1002/num.20573, 2010.

[17] M. A. Ramadan, K. R. Raslan, M. A. Nassar, An approximate analytical solution of higher order linear differential equations with variable coefficients using improved rational Chebyshev collocation method, Appl. Comput. Math. 3(6) (2014) 315-322. 